Dear author,

Please note that changes made in the online proofing system will be added to the article before publication but are not reflected in this PDF.

We also ask that this file not be used for submitting corrections. 


\title{
Evaluating thermoacoustic properties of heating appliances considering the burner and heat exchanger as acoustically active elements
}

\author{
Naseh Hosseini ${ }^{a, b, *}$, Viktor Kornilova ${ }^{a}$ O.J. Teerling ${ }^{b}$, Ines L. Arteaga ${ }^{a, c}$, L.P.H. de Goey ${ }^{a}$ \\ a Mechanical Engineering Department, Eindhoven University of Technology, P.O. Box 513, Eindhoven 5600 MB, The Netherlands \\ ${ }^{b}$ Bekaert Combustion Technology B.V., J.C. van Markenstraat 19, Assen 9403 AR, The Netherlands \\ ${ }^{\mathrm{c}}$ Department of Aeronautical and Vehicle Engineering, KTH Royal Institute of Technology, Stockholm SE-100 44, Sweden
}

\section{A R T I C L E I N F O}

\section{Article history:}

Received 27 June 2017

Revised 20 January 2018

Accepted 21 January 2018

Available online xxx

\section{Keywords:}

Thermoacoustic

Combustion instability

Laminar flame

Heat exchanger

Transfer function

Transfer matrix

\begin{abstract}
A B S T R A C T
Heat exchangers are an essential constituent part of many combustion systems. The thermoacoustic instability in such systems is a common problem and it has been studied extensively. However, the heat exchanger has not gained much attention in the field of combustion thermoacoustics, leading to a lack of knowledge about the thermoacoustic interactions between the burner and the heat exchanger. In this paper, a modeling approach is introduced to study these interactions in an academic representation of a heating appliance, comprised of a perforated slit burner and a tube heat exchanger. Both elements are considered thermally and acoustically active. A CFD model is used in a two-dimensional domain to simulate the response of the system to small amplitude broadband velocity perturbations. The thermochemical and acoustic coupling between the burner and the heat exchanger is investigated and a method is introduced to decouple their effects and study them separately. The extents to which this method is valid are addressed by varying the distance between the elements. Results show that as long as the flames do not impinge on the heat exchanger surface, a linear network modeling approach can be applied to construct the acoustic response of the composed configuration from the responses of its constituting elements. This approach requires registering the average velocity on a properly chosen intermediate plane between the burner and heat exchanger. Choosing this plane may be to some point difficult, i.e. when the burner and heat exchanger are close and cannot be considered independent. Moreover, when flame impingement occurs, the interactions between the flame and heat exchanger affect their individual thermoacoustic behaviors and the burner plus heat exchanger assembly needs to be considered as one coupled acoustic element. Particularly, flame impingement changes the phase of the heat absorption response of the heat exchanger and it may significantly alter the acoustic properties of the coupled assembly. The physics lying behind the effects of such interactions on the thermoacoustics of the system is discussed. The obtained results signify that a correct stability prediction of an appliance with burner and heat exchangers requires considering active thermoacoustic behavior of both elements as well as their interactions.
\end{abstract}

(c) 2018 The Combustion Institute. Published by Elsevier Inc. All rights reserved.

\footnotetext{
* Corresponding author.

E-mail address: n.hosseini@tue.nl (N. Hosseini).
}

solely active thermoacoustic element and the other components as passive elements (such as ducts, vessels and terminations, including heat exchangers) [4]. One of the crucial prerequisites of this modular method is the possibility to distinguish and separate the "acoustic elements" from each other, and accordingly to encapsulate their acoustic behavior as a property of the given element only. This paradigm is the basis of the network model approach, where the subsystems are described as linear elements with two acoustic inputs and two outputs (two-ports), interrelated via a Transfer Matrix (TM) or a Scattering Matrix (SM). The heat release response to acoustic excitation of velocity in an active element, like burner, can be naturally described within the concept of the thermoacoustic Transfer Function (TF). For acoustically compact 
elements (where the size of the element is negligible compared to the acoustic wavelength), the $T M$ is related to the $T F$ through the heat release rate $[5,6]$. There are multiple examples in the literature, where this approach has proven to be productive in predicting system instabilities and evaluating different measures of passive and/or active control [7-10].

On the system level, the heat exchanger is designed to absorb most of the heat produced by the burner and create a reverse temperature jump of the same order of magnitude as the burner. On the other hand, it is well known that an acoustically forced flow may create a fluctuating heat flux on the bodies in the flow [11,12]. Therefore, the heat exchanger may potentially be an additional active acoustic element next to the burner in the acoustic network. The activity of a heat exchanger can be included in the acoustic network analysis. As an example, the authors have demonstrated in an earlier publication that in a simple one-dimensional Rijke burner with a flame and heat exchanger, the thermoacoustic properties of the heat exchanger can significantly alter the stability of the system [13]. However, the aforementioned study has been purely acoustic and considered approximated and frequency independent transfer functions for the burner and heat exchanger, neglecting any direct interactions.

On the other hand, the practical need to reduce the built volume of combustion appliances and minimize nitrogen oxides, leads to the desire to locate the heat exchanger very close to the burner. The smaller this distance, the more questionable it becomes to consider the burner and the heat exchanger as two acoustically independent elements. It is known that in the limit case of impinging flames, the intense interaction of a flame with a heat exchanger leads to significant changes of the acoustic properties of the flame. Particularly, it was observed that impingement may change the noise produced by the flame [14-16]. Similarly, the proximity of a flame to the heat exchanger surface can affect the periodic heat transfer between the surface and hot gases. In other words, the fluctuations induced by the flame dynamics may alter the acoustic properties of the heat exchanger. Consequently, the thermoacoustic properties (e.g. TF and TM) of the combination of the burner and heat exchanger may differ drastically from when considered independent. Therefore, one may a-priori expect that the mutual interactions between the burner and the heat exchanger may alter their individual thermoacoustic properties. Consequently, the direct interactions (hydrodynamic, heat transfer and chemical reactions) will impose a range of implications for the acoustic network modeling of a combustion system with interacting burner and heat exchanger.

The practical relevance, fundamental interest and shortage of knowledge about the burner-heat-exchanger thermoacoustic interactions has stimulated the present research. The ultimate goal of this investigation is to elucidate the physical nature of burner-heatexchanger interactions in respect to their thermoacoustic behavior. Particularly, the aim is to answer the following questions:

- What are the conditions for considering the combined TF/TM of the burner and heat exchanger as the superposition of their individual TF/TM's, and how can this be done properly?

- When do the interactions between them affect the individual TF/TM's, and what is an applicable method to reconstruct a combined TF/TM?

- What are the physical phenomena and governing parameters responsible for these interactions?

To solve the formulated problem and questions, a particular configuration is studied that consists of a bed of idealized Bunsentype wedge shaped (two-dimensional) premixed flames anchored on slot perforations in a burner deck, and interacting with a heat exchanger consisting of a series of idealized cylindrical tubes placed downstream the burner (see Fig. 1). This academic configu- ration is representative for the design of a majority of heating appliances and is composed of relatively simple elements which have been intensively studied in the past on theoretical, experimental and numerical levels [12,17-19].

CFD is chosen as the research tool because there are no convenient and easily measurable indicators for the oscillating rate of heat transfer from the hot gases to the heat exchanger (unlike the chemiluminescence of chemical radicals for flame heat release). Consequently, within a physical experiment, it is feasible to evaluate the effects of the heat exchanger on the acoustic properties of the flame, but it is difficult to detect the reciprocal effects of the flame on the heat exchanger. This fact motivates the use of the CFD-based analysis within the present research.

By changing the distance between the burner and heat exchanger, one can alter the intensity of the mutual interactions. For large distances, no hydrodynamic interaction is expected. However, as the distance decreases the hydrodynamic and thermal fields created by the flame and heat exchanger begin to interact. These interactions can be extremely intense and complicated when the flame impinges on the heat exchanger surface. Details of the situations that may occur are discussed in the following section.

Ultimately, by studying this simplified configuration, general conclusions are formulated regarding the physics of the processes responsible for the interactions, considering the thermoacoustic aspects of this problem. The drawn conclusions are generic by nature and can be applicable to other configurations as well.

\section{Governing phenomena of the interactions between a burner and a downstream placed heat exchanger}

A picture of the constructed burner-heat-exchanger system and a schematic of the simplified configuration used as the simulation geometry are illustrated in Fig. 1. Symmetry has been used in order to reduce the size of the computational domain and to model the effects of the neighboring flames and heat exchanger tubes. The mixture enters from the inlet at the bottom and the flames stabilize on the slit perforations. The combustion products flow toward the heat exchanger tubes and leave the domain at the outlet. In addition to the dimensions mentioned in Fig. 1, other important length scales are flame height ( $L \approx 5 \mathrm{~mm}$ ), flame thickness $(\delta \approx 0.5 \mathrm{~mm})$ and the smallest acoustic wavelength $(\lambda \approx 1370 \mathrm{~mm})$, which is calculated for a maximum temperature of $2000 \mathrm{~K}$ and highest relevant frequency of $1000 \mathrm{~Hz}$.

A-priori, for a heating appliance containing a burner and heat exchanger and in presence of acoustic perturbations, one can foresee several hypothetical interaction scenarios between the two elements. These interactions are mainly governed by the distance between them and are illustrated in Fig. 2. This figure shows the flow streamlines and the flame shape. In the limit case where the distance is sufficiently large to smoothen all non-uniformities (thermal, kinetic and fluid dynamic), the heat exchanger is exposed to a uniform flow of burned gases with the acoustic perturbations on top of the mean flow (see Fig. 2a). In this case, the subsystems can be treated separately from the point of view of either convective fluid dynamics or acoustics. In this case, there are no difficulties to define spatial boundaries as inlets and outlets for the burner and the heat exchanger as the succeeding element. Usually these boundaries are defined where acoustic waves can be considered one-dimensional and planar. It can even be necessary and reasonable to include some extra acoustic elements, such as ducts, to model the propagation of acoustic waves between the burner and the heat exchanger [20].

At some level of proximity between the subsystems, either the convective or the acoustic perturbations do not smoothen to a negligible level (see Fig. 2b). In this case, the thermoacoustic behavior of the heat exchanger may be affected due to the deviations of 
a
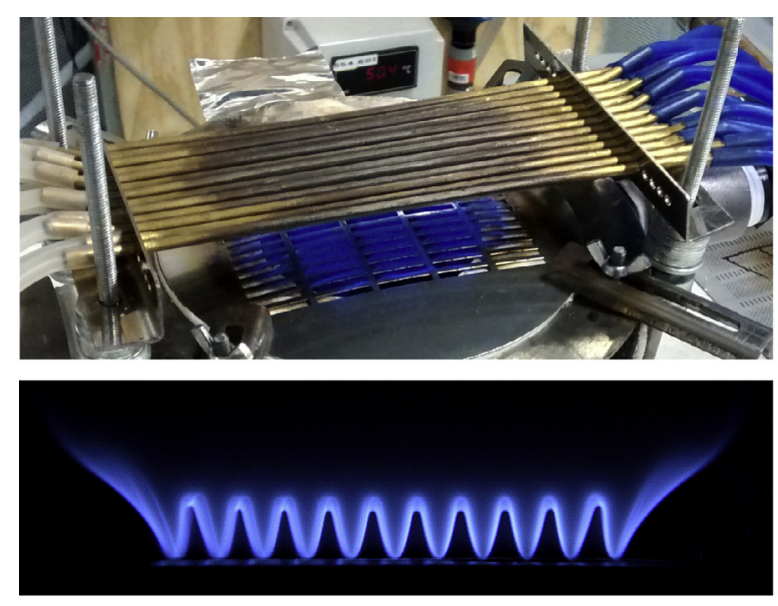

b

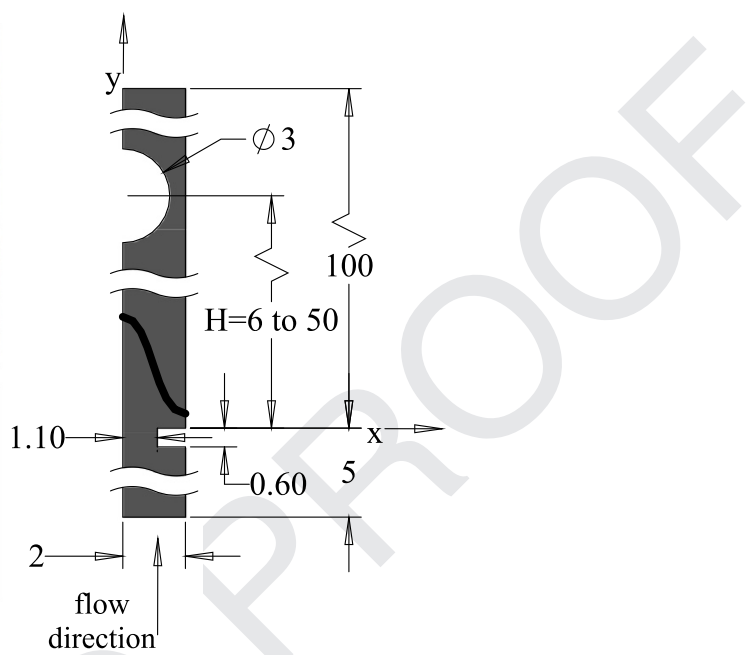

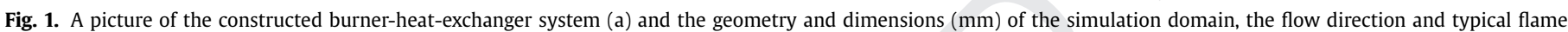
shape (b).

the incoming fluid field. This is the situation where the properties of the flame are not yet affected by the heat exchanger, but the acoustic properties of the heat exchanger are affected by the proximity of the flame. In general, the convective and acoustic "nearfields" of the excited flame do not have to be spatially the same. Consequently, within this one-way interaction scenario some subcases can be foreseen based on the intensity of the effects of the burner on the heat exchanger. Furthermore, the existence of an acoustically well-defined interface between the burner and heat exchanger is questionable.

Finally, when the heat exchanger is so close to the burner that the mean flame shape is affected, mutual thermoacoustic interactions are expected (see Fig. 2c). Here, the subsystems may no longer be acoustically separated and should be considered as one combined element with common inlet and outlet interfaces.

Within the present contribution, the above-described scenarios are studied in the presented setup. First, the numerical setup is introduced concisely together with brief information about model validation. More details on the model and its validation approach can be found in a previous publication [21]. Next, the thermoacoustic properties of the burner and heat exchanger, and their combined TFs and TM's are studied as functions of the distance between the burner surface and the heat exchanger. This is the core analysis, which is meant to reveal the change of the interaction scenarios. The observed interactions are then analyzed in the discussion section, and in conclusions, the generic features of the interactions between the burner and the heat exchanger are retrieved based on the obtained results.

\section{Numerical setup}

The simulation domain is presented in Fig. 1. The inlet boundary is set to constant velocity of $0.8 \mathrm{~m} / \mathrm{s}$, the outlet boundary to atmospheric pressure and the lateral boundaries to symmetric. Some studies have suggested using a correction factor for flame expansion effects [22]. However, these effects marginally alter the flame TF and do not change the nature of the interactions and the conclusions to be drawn. The burner deck and heat exchanger have isothermal boundaries set to 793 and $343 \mathrm{~K}$, respectively, obtained from corresponding measurements, where the walls are thin and the constant temperature assumption is acceptable. Nevertheless, if a more accurate and detailed investigation is required, conjugate heat transfer needs to be taken into account. The grid size is $160 \mu \mathrm{m}$, which is refined to 40 and $20 \mu \mathrm{m}$ around the heat exchanger and flame, respectively, in order to resolve the thermal boundary layers and flame thickness properly.

The flow is assumed laminar and the fluid an incompressible ideal gas, due to low Reynolds and Mach numbers. The mixture is methane-air with the equivalence ratio of 0.8 and combustion is modeled using the finite rate chemistry for a 2-step reaction mechanism as described in [23]. The available Arrhenius rates are modified to match the flame speed and thickness to the available experimental data (see Section 4). The specific heats of all individual species are obtained from temperature-dependent polynomials and their viscosities and thermal conductivities using kinetic theory. The corresponding properties of the mixture are then available using the mixing law. The software package used for this work is ANSYS ${ }^{\circledR}$ Fluent, Release 17.0.

The transfer function concept is widely used to characterize the thermoacoustic properties of active elements driven by acoustic velocity in the linear regime. In the frequency domain, the frequency-dependent $T F$ is defined as the ratio of the relative perturbation of heat release or absorption, to the relative perturbation of flow velocity,

$T F(f)=\frac{q^{\prime}(f) / \bar{q}}{u^{\prime}(f) / \bar{u}}$

where $q$ is the heat release or absorption, $u$ the flow velocity and $f$ the frequency of the perturbation. The prime and overbar denote the fluctuating and mean parts of the variables, respectively.

A purely acoustical concept to characterize the thermoacoustic properties of an element is the transfer matrix, in which part of the system is considered as a lumped acoustic element. This approach is most suitable to describe longitudinal acoustic waves in the linear regime of oscillations [3]. The input vector of acoustic variables can be related to the output vector via the transfer matrix,

$$
\left[\begin{array}{l}
p^{\prime} \\
u^{\prime}
\end{array}\right]_{\text {downstream }}=T M(f)\left[\begin{array}{l}
p^{\prime} \\
u^{\prime}
\end{array}\right]_{\text {upstream }}, T M(f)=\left[\begin{array}{ll}
M_{p p}(f) & M_{p u}(f) \\
M_{u p}(f) & M_{u u}(f)
\end{array}\right],
$$

where $p^{\prime}$ and $u^{\prime}$ denote the acoustic pressure and velocity, respectively. It is known that for an acoustically compact element in the limit of low Mach numbers, $M_{u u}$ is the only entry that is sensitive to the presence of an oscillating heat source or sink [22,24-26]. 

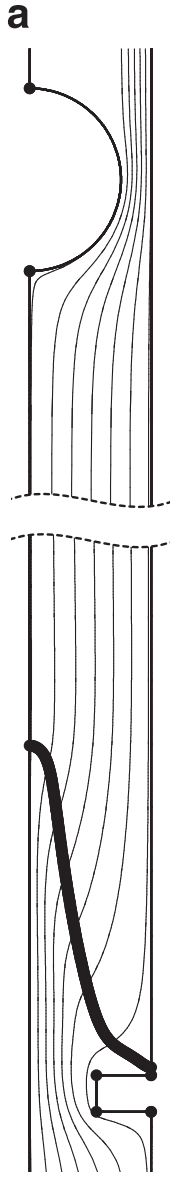

b

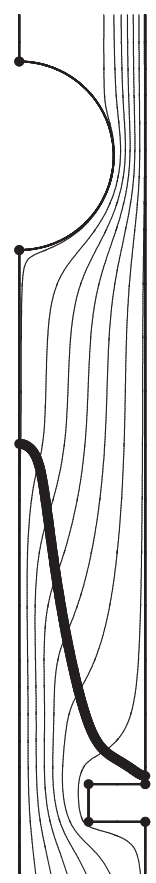

C

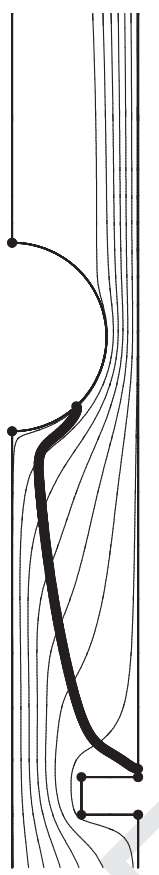

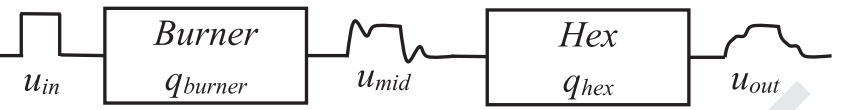

Fig. 3. A schematic of the network model for the total system showing the excitation and response signals.

Table 1

The excitation and response signals used for calculating the $T F$ and $T M$ of each element and the total system.

\begin{tabular}{lll}
\hline Parameter & Excitation & Response \\
\hline$T F_{\text {burner }}$ & $u_{\text {in }}$ & $q_{\text {burner }}$ \\
$T F_{\text {hex }}$ & $u_{\text {mid }}$ & $q_{\text {hex }}$ \\
$T F_{\text {total }}$ & $u_{\text {in }}$ & $q_{\text {burner }}-q_{\text {hex }}$ \\
$T M_{\text {burner }}$ & $u_{\text {in }}$ & $u_{\text {mid }}$ \\
$T M_{\text {hex }}$ & $u_{\text {mid }}$ & $u_{\text {out }}$ \\
$T M_{\text {total }}$ & $u_{\text {in }}$ & $u_{\text {out }}$ \\
\hline
\end{tabular}

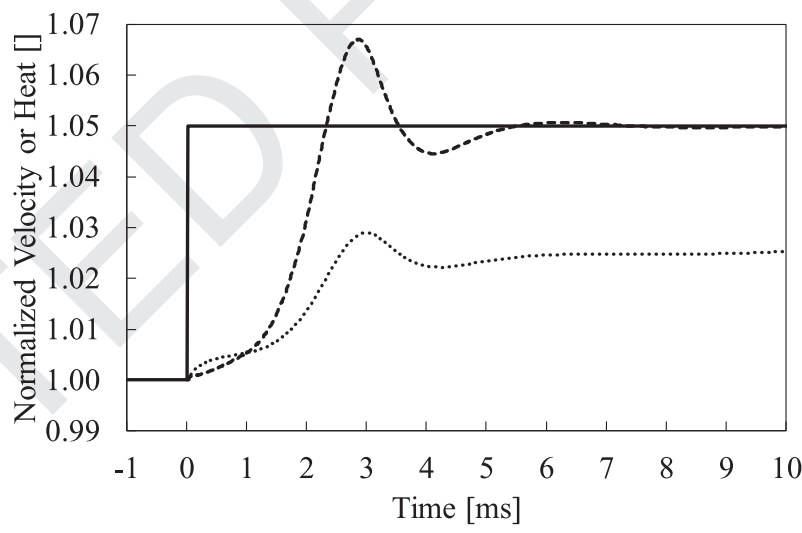

Fig. 4. Normalized time signals of the inlet velocity (_ ${ }_{-}$), burner heat release (---) and heat exchanger heat absorption (.......). The normalization is performed with respect to corresponding values before excitation.

Fig. 2. The flow streamlines and flame shapes for various conditions of interaction between the burner and heat exchanger, i.e. flame and heat exchanger unaffected (a), flame unaffected and heat exchanger affected (b) and both flame and heat exchanger affected (c).

This entry relates the acoustic components of the velocity downstream and upstream of an element, where the acoustic fields are planar. For the sake of simplicity, the $T M_{22}$ entry of the transfer matrix $\left(M_{u u}\right)$ is referred to as $T M$ in the rest of the article. The relation between TF and TM can be derived from the RankineHugoniot jump conditions [27] and shows their linear proportionality,

$T M=1+(\theta-1) T F$,

where $\theta$ is the ratio of downstream to upstream temperatures in Kelvins.

In order to obtain the burner and heat exchanger $T F$ or $T M$, transient simulations are performed to calculate their responses to a block change in velocity. This is done by imposing a step function with $5 \%$ increase in the inlet velocity as excitation. The response of the burner and heat exchanger are then calculated using the volume integral of reaction source term and the surface integral of heat flux through the heat exchanger wall, respectively. It is particularly checked that the time step size $(10 \mu \mathrm{s})$ is small enough to capture the premixed flame dynamics. It is also checked that the perturbations are small enough to prevent nonlinearities and can be regarded as a broadband excitation covering a wide range of frequencies. This approach has proven to be efficient and representative of obtaining the system response using discrete harmonic excitations [28]. The responses of the system and its elements are then calculated both with the transfer function and transfer matrix approaches, according to Fig. 3 and Table 1.
In Fig. 3, a schematic of the system as a network model is shown, where $u$ and $q$ denote velocity and heat release/absorption, respectively. Note that the reference point for calculating $T F$ of a specific element is usually chosen at a distance upstream of the element, where the acoustic waves can be assumed planar. Consequently, for the burner, the combined effects of the flame and flame holder are measured or simulated.

Figure 4 shows how the burner heat release and heat exchanger heat absorption change with time as the velocity excitation is applied. This data is for a case where the heat exchanger is placed far from the burner (see Fig. 2a).

Table 1 summarizes the corresponding excitation and response signals used for calculating the TF and TM of each element and the combined system.

\section{Validation}

The modified reaction parameters are first used in a onedimensional configuration to find the grid size required to capture the flame thickness and laminar flame speed accurately, as well as their dependency on the equivalence ratio and unburnt temperature. The obtained data are in good agreement with the literature [29-31]. In addition, the validation of the TF of the twodimensional stretched flame is performed in comparison with the work of Kornilov et al. [32]. They have provided measurement and DNS simulation results of wedge flames stabilized on a perforated deck. This work is chosen because the configuration is close to our study and their results are confirmed in other independent studies as well [22]. The comparison of the TF gain and phase is presented 

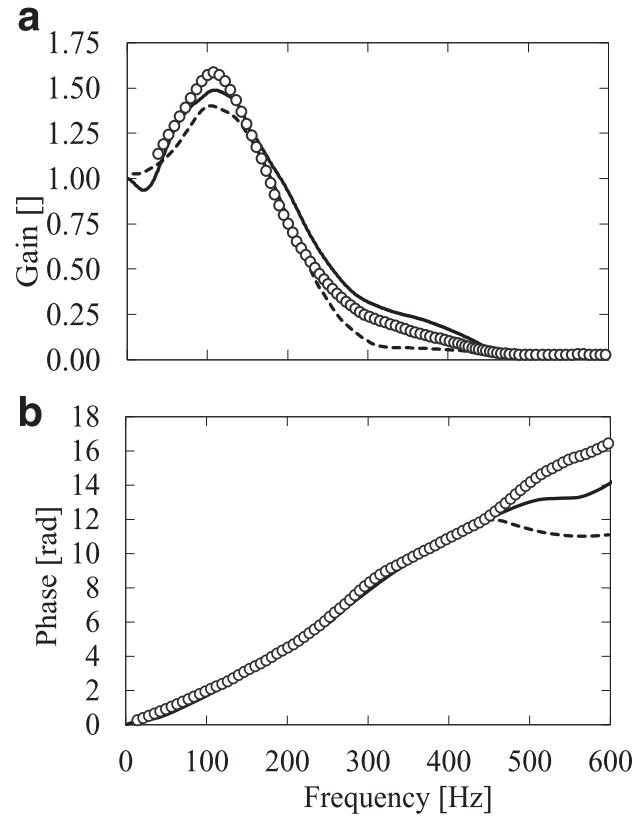

Fig. 5. The gain (a) and phase (b) of the burner $T F$ for the experiments from [32] (O), simulations from [32] (_-_) and this study (_ $)$.

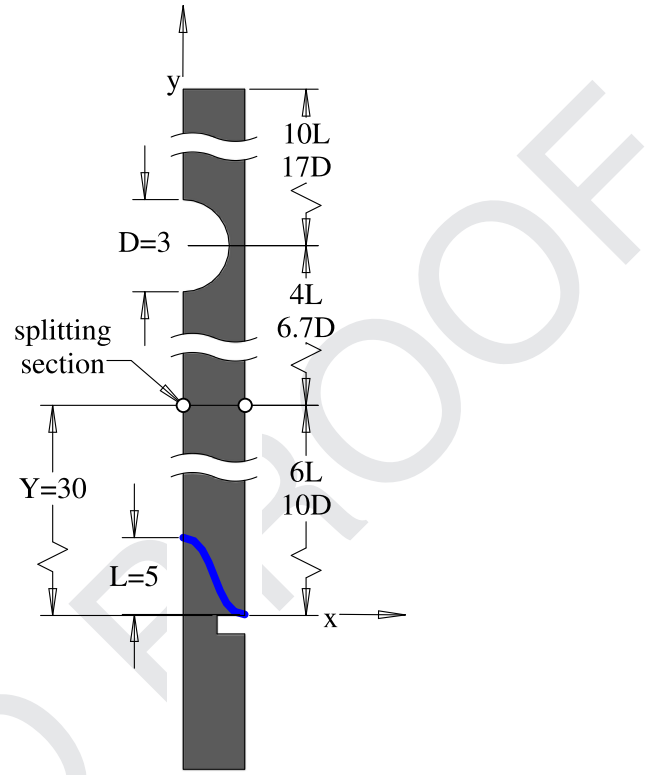

Fig. 6. Schematic of the split and related dimensions ( $\mathrm{mm})$.

The total TF can be calculated theoretically using the energy balance in the system and it is not simply the sum or a weighted sum of the burner and heat exchanger TF's. Assuming linearity of the system and using the heat balance we have,

$q_{\text {total }}=q_{\text {burner }}-q_{\text {hex }} \Rightarrow\left\{\begin{array}{l}\bar{q}_{\text {total }}=\bar{q}_{\text {burner }}-\bar{q}_{\text {hex }} \\ q_{\text {total }}^{\prime}=q_{\text {burner }}^{\prime}-q_{\text {hex }}^{\prime}\end{array}\right.$.

Based on the definition of $T F$ and some algebraic manipulations we can calculate,

$T F_{\text {total }}=\left(\frac{\bar{q}_{\text {burner }}}{\bar{q}_{\text {total }}}\right) T F_{\text {burner }}-\left(\frac{\bar{q}_{\text {hex }}}{\bar{q}_{\text {total }}}\right) T F_{\text {hex }} T F_{u_{\text {mid }}-u_{\text {in }}}$.

Here $T F_{u_{\text {mid }}-u_{\text {in }}}$ is a velocity-to-velocity transfer function between the inlet velocity and the velocity downstream of the flame (upstream of the heat exchanger). It can be related to burner TM using the velocity ratio $u_{\text {in }} / u_{\text {mid }}$. This ratio is equal to $1 / \theta$ for an incompressible ideal gas. Therefore, using Eq. (3) we have,

$$
\begin{aligned}
T F_{u_{\text {mid }}-u_{i n}} & =\left(\frac{\bar{u}_{\text {in }}}{\bar{u}_{\text {mid }}}\right) T M_{\text {burner }}=\left(\frac{1}{\theta}\right)\left(1+(\theta-1) T F_{\text {burner }}\right) \\
& =\frac{1}{\theta}+\frac{\theta-1}{\theta} T F_{\text {burner }},
\end{aligned}
$$

so that we have,

$T F_{\text {total }}=\left(\frac{\bar{q}_{\text {burner }}}{\bar{q}_{\text {total }}}\right) T F_{\text {burner }}-\left(\frac{\bar{q}_{\text {hex }}}{\bar{q}_{\text {total }}}\right) T F_{\text {hex }}\left(\frac{1}{\theta}+\frac{\theta-1}{\theta} T F_{\text {burner }}\right)$.

Figure 7 illustrates the gain and phase of the TF of the burner, 326 heat exchanger and total system using both the CFD results and 327 Eq. (4). Here, the heat exchanger TF is calculated using the veloc- 328 ity fluctuations at the splitting section. The gain of the burner TF 329 has an overshoot at $300 \mathrm{~Hz}$ which is due to the coupling of pe- 330 riodic heat transfer to the burner deck with the velocity fluctua- 331 tions [17]. The TF phase, and consequently the time delay, of the 332 burner is much larger than that of the heat exchanger and there- 333 fore the total system time delay is almost identical to that of the 334 burner. The gain of the heat exchanger $T F$ is below unity for all 335 frequencies, i.e. the relative amplitude of fluctuations of the heat in Fig. 5. This comparison also provides a double check for the laminar flame speed and flame height, since these parameters significantly affect the burner $T F[22,26,32]$. The results show that the model is able to reproduce the experimental and numerical data from the literature with good agreement.

\section{Results and discussion}

The results are categorized in two parts. First, the case where the heat exchanger is placed far downstream the burner is investigated. Here the sensitivity of the location of the chosen section for decoupling the burner and heat exchanger is studied. Afterwards, various situations that occur when the physical distance between the flame and heat exchanger decreases are discussed.

\subsection{The heat exchanger far downstream the burner (at $\mathrm{Y}=50 \mathrm{~mm}$ )}

As can be noticed in Fig. 2, the flow accelerates across the flame due to the temperature increase. Since the configuration is confined, a velocity profile downstream the flame is formed which is not flat (not constant in $\mathrm{x}$-direction). The required distance from the burner so that this velocity profile is flat with a maximum deviation of $1 \%$ is defined by $Y=0.05 . R e^{\prime} . s$, where $s$ is the pitch between the slits ( $4 \mathrm{~mm}$ in Fig. 1b) and $R e^{\prime}$ is the Reynolds number (based on the velocity downstream the slits and two times the pitch) [33]. This minimum length is calculated equal to $30 \mathrm{~mm}(6 \mathrm{~L})$ for the geometry and conditions under investigation. This location $(Y=30 \mathrm{~mm})$ is $20 \mathrm{~mm}(>6 D)$ upstream of the heat exchanger tube, which is far enough for the flow not to be affected by the contraction between the tubes. Therefore, this location is chosen as a mid-section for splitting the system into the burner and heat exchanger subsystems, when they are far from each other. Figure 6 shows a schematic of the splitting section and related dimensions.

The Helmholtz number is described as $H e=2 \pi l / \lambda$, where $l$ is the corresponding length and $\lambda$ is the acoustic wavelength [34]. For $30 \mathrm{~mm}$ length and the smallest acoustic wavelength of $\lambda=1370 \mathrm{~mm}$ (see Section 2), the Helmholtz number is less than 0.14 . This shows that the subsystems after splitting are still acoustically compact. 


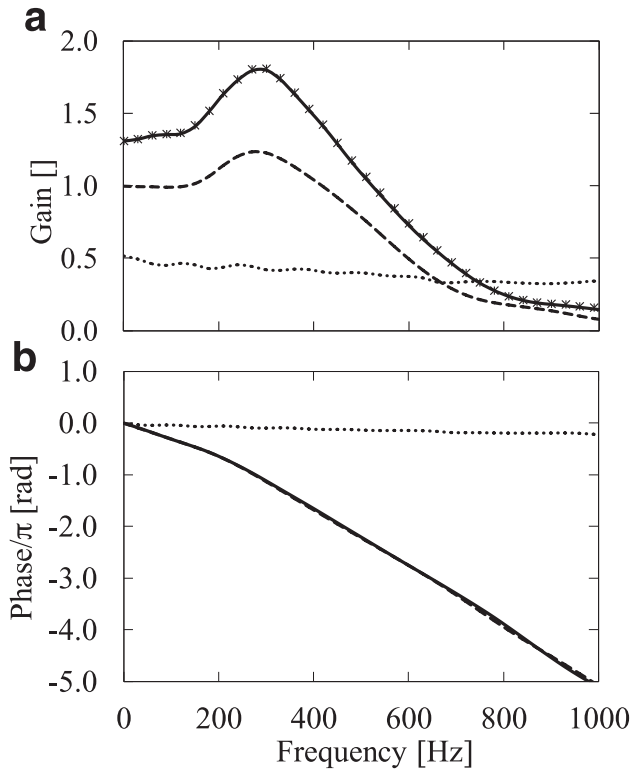

Fig. 7. The gain (a) and phase (b) of the $T F$ of the total system via CFD (_ $)$, via Eq. (4) (X), burner (.--) and heat exchanger (......) when it is placed at $Y=50 \mathrm{~mm}$.

flux through the heat exchanger is smaller than that of the excitation. This is a usual trend in bluff bodies exposed to oscillating cross flow [11,12]. Since the heat exchanger is a sink of heat, this causes the relative amplitude of fluctuations of the total heat of the whole system to be larger than that of the excitation, resulting in larger gain for the total TF. This causality can be understood by considering harmonic fluctuations with DC and AC components of the signal. In an extreme case, in which the gain of the heat exchanger $T F$ is very small, the heat exchanger is still absorbing heat as a mean value (DC component), but the fluctuating part (AC component) of the total heat is still almost equal to that of the burner, since the heat exchanger absorption has negligible fluctuations. Therefore, the ratio of total system heat fluctuations to its mean is always larger than that of the burner. This can also be understood using the data presented in Fig. 4 . If the $5 \%$ increase in the excitation signal had led to $5 \%$ increase in burner heat release and $5 \%$ increase in heat exchanger heat absorption, then the total system heat would have also increased by $5 \%$. However, the heat exchanger absorption has only increased by $2 \%$, which means that the total heat of the system (burner minus heat exchanger) has increased more than $5 \%$. This causes a gain of the total TF to be larger than one. Note that this behavior may change depending on the constructive or destructive behaviors due to the changes of $T F$ phase for various frequencies.

Figure 8 shows the gain and phase of TM for the burner, heat exchanger and total system. Since $T M$ is relating the velocities upstream and downstream an element (dilatation rate), its gain is larger than one if the temperature increases (expansion) and smaller than one if it decreases (contraction). This is clearly visible in Fig. 8. The TM and TF are related via Eq. (3) and depending on the values of $T F$ and $\theta, T M$ may fluctuate with frequency. This can be seen in the form of fluctuations in the gain of $T M$ in Fig. 8. These fluctuations are also physically important since they illustrate that the information obtained directly from the $T F$ does not suffice for judging the system dilation rate, which is important when the system is exhibiting Helmholtz oscillations. While the dilatation rate is around six (the steady state value due to the temperature jump) at $400 \mathrm{~Hz}$, it reduces to one (transparent element) slightly above $600 \mathrm{~Hz}$. The phase of $T M$ of the total system

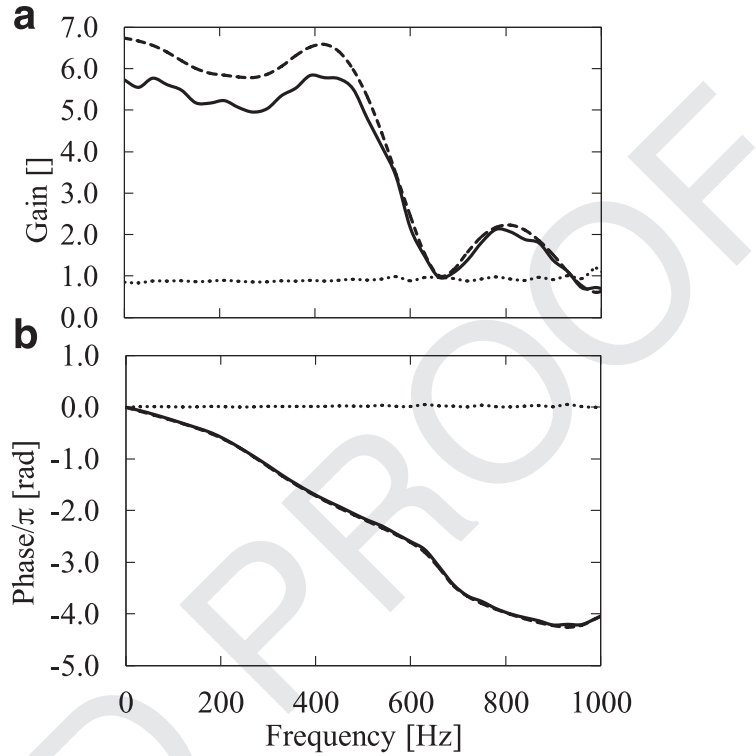

Fig. 8. The gain (a) and phase (b) of the $T M$ of the total system (_ and heat exchanger (......) when it is placed at $Y=50 \mathrm{~mm}$.

follows that of the flame for the same reason mentioned for TFs 376 (see the explanation of Fig. 7).

\subsection{Varying the location of the splitting section}

The calculations for the case with the heat exchanger far downstream the burner are straightforward, however they become more complicated when the heat exchanger is placed closer to the burner. The first step toward bringing the heat exchanger closer to the burner is to keep the heat exchanger location fixed, but vary the location of the splitting section. In order to study the response of the heat exchanger under these conditions, the splitting section is moved from its previous position $(Y=6 \mathrm{~L}=10 \mathrm{D}=30 \mathrm{~mm})$ to further upstream $(Y=1.4 L=2.33 \mathrm{D}=7 \mathrm{~mm})$ and downstream $(Y=9.2 \mathrm{~L}=15.67 \mathrm{D}=47 \mathrm{~mm})$ and its response is investigated. The flame height is $L=5 \mathrm{~mm}$ and the heat exchanger diameter is $D=3 \mathrm{~mm}$ and is placed at $Y=50 \mathrm{~mm}$, therefore, $Y=7$ and $47 \mathrm{~mm}$ correspond to $2 \mathrm{~mm}$ downstream the flame tip and $1.5 \mathrm{~mm}$ upstream the heat exchanger surface, respectively. This is the closest possible to the flame tip and heat exchanger surface, without interfering with the temperature boundary layers.

In Fig. 9, the changes of velocity magnitude with time at these sections are shown. At each section, the transient velocity magnitude is plotted at the point where $X=0 \mathrm{~mm}$ (the left symmetry line) in dashed lines, at $X=2 \mathrm{~mm}$ (the right symmetry line) in dotted lines, and the average velocity on the section in solid lines. The two points in x-direction are shown on the splitting section in Fig. 6 and the values are normalized to their corresponding initial values before the $5 \%$ step excitation. At each section, if the velocity profile preserves its shape with time (stays self-similar), the normalized velocity at all the points in X-direction will plot on each other in Fig. 9. This is the case for $Y=30$ and $47 \mathrm{~mm}$, however for $Y=7 \mathrm{~mm}$ there is a variation, which is explained later. Despite this variation, the average value on the splitting section (solid lines in Fig. 9) has almost identical transient behavior for all the sections. This means that the $\mathrm{x}$-averaged value at any of these sections (even the ones extremely close to the flame tip or heat exchanger surface) or any other section between them may be used for splitting the system into the burner and heat exchanger sub elements.

Figure 10 better illustrates the self-similarity of velocity profiles with time. The non-normalized velocity magnitudes along the
379 380 381 
a

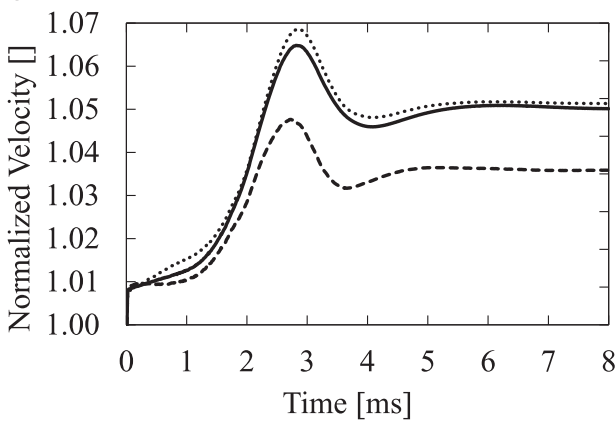

b

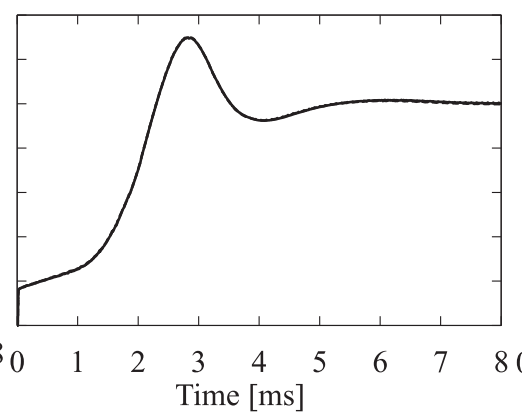

C

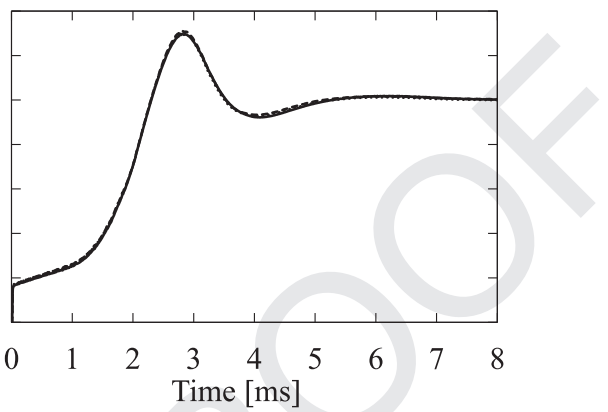

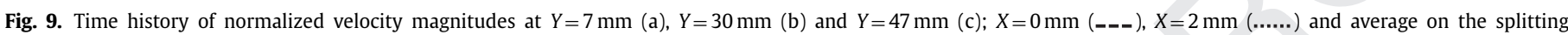
section (—); the heat exchanger is placed at $Y=50 \mathrm{~mm}$.

a

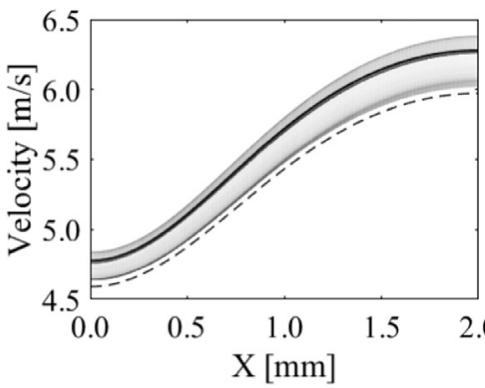

b

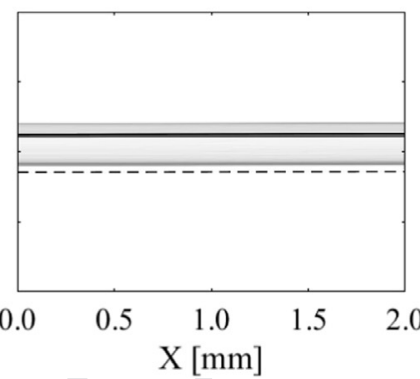

C

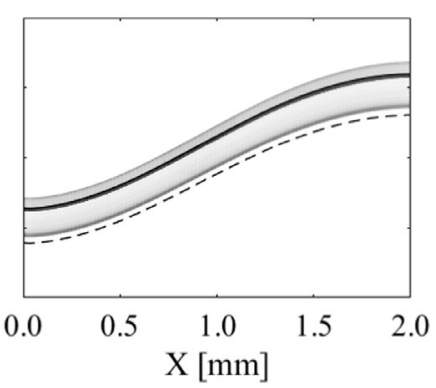

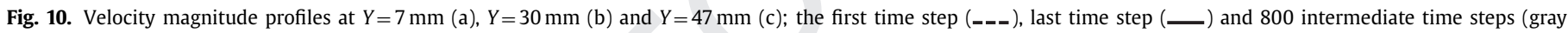
shaded lines).

$\mathrm{x}$-direction are plotted for the first time step (just before applying the excitation) in dashed lines, for the last time step in solid lines and for 800 intermediate time steps $(8 \mathrm{~ms})$ in semi-transparent gray lines, forming the gray shaded area. For $Y=7$ (Fig. 10a) and $47 \mathrm{~mm}$ (Fig. 10c), it can be observed that the non-uniform velocity profiles are induced by the flow acceleration through the flame and contraction between the heat exchanger tubes, while the velocity profile at $Y=30 \mathrm{~mm}$ remains flat. The velocity profiles at $Y=30$ and $47 \mathrm{~mm}$ stay self-similar with time, however, at $Y=7 \mathrm{~mm}$ the expansion caused by the flame grows in $x$-direction, i.e. the increase in velocity is smallest at $X=0 \mathrm{~mm}$ and largest at $X=2 \mathrm{~mm}$ (see Fig. 10a). This was previously seen in Fig. 9a as the difference between the dashed and dotted lines, and is because the flow expansion through the flame front is weaker at the flame tip $(X=0 \mathrm{~mm})$.

The gain and phase of the heat exchanger $T F$ using the $x$ averaged velocities at the three sections are plotted in Fig. 11. The markers show the results for the "NoFlame" case, i.e. the case where no flame is simulated and the heat exchanger is only exposed to a fluctuating flow with post-combustion properties (mean velocity, composition and temperature). It is evident that choosing different heights for calculating the heat exchanger transfer function leads to almost the same results, which are all in acceptable agreement with the NoFlame case. The observed fluctuations in the gain and phase are because in the NoFlame case, the excitation signal is a perfect step function, while for the other cases the measured velocity at the sections are used as excitation and this measured velocity is not a perfect step. This poses an imperfect excitation signal that may lead to numerical errors. In fact if a step function is not possible as excitation (for example in measurements), a separate study needs to be performed to find a proper excitation signal. Details of creating such signals can be found in the literature [35]. These results prove that the discussed decou-

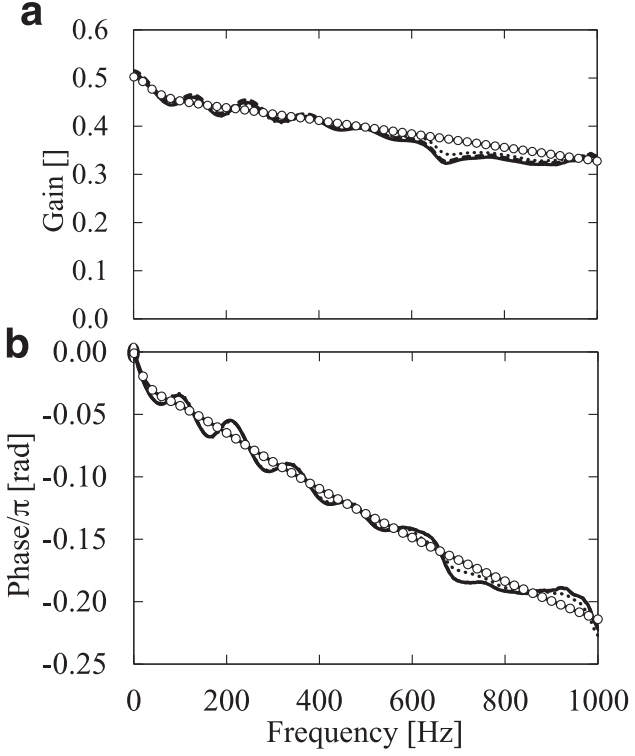

Fig. 11. The gain (a) and phase (b) of the heat exchanger $T F$ when it is placed at $Y=50 \mathrm{~mm}$ with respect to $\mathrm{X}$-averaged velocity at $Y=7(\ldots . .),. 30(-\mathbf{c})$ and $47 \mathrm{~mm}$ $(-)$, and the NoFlame case (-O-).

pling method is valid even when the heat exchanger is exposed to a non-flat velocity profile.

5.3. Varying the distance between the burner and the heat exchanger 450

Decreasing the distance between the burner and heat exchanger 451 initially exposes the heat exchanger to a non-flat velocity profile, 452 

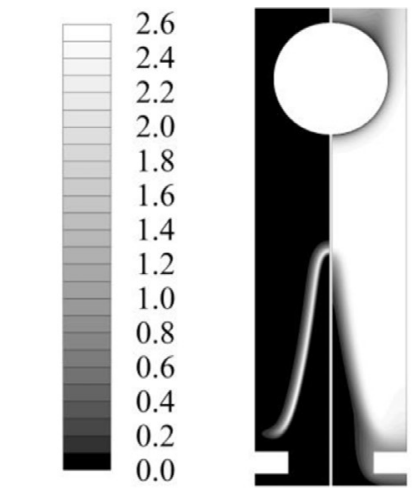

Reaction Heat [W] $\mathrm{Y}=10 \mathrm{~mm}$

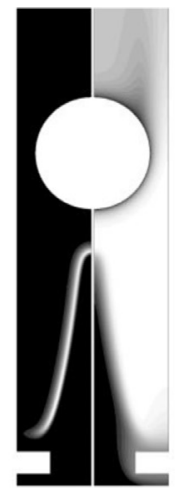

$\mathrm{Y}=8 \mathrm{~mm}$

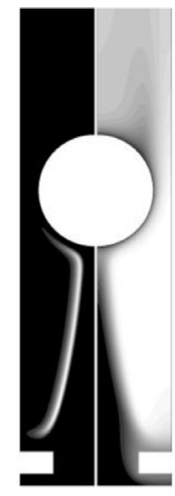

$\mathrm{Y}=7 \mathrm{~mm}$
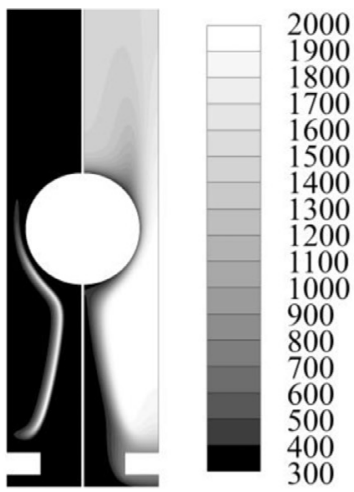

$\mathrm{Y}=6 \mathrm{~mm}$

Temperature [K]

Fig. 12. Contours of reaction heat (W) (left sides) and temperature (K) (right sides) for the cases with the heat exchanger at $Y=10,8,7$ and $6 \mathrm{~mm}$.

then a non-flat temperature and/or concentration profile, and finally flame quenching and different impingement regimes occur. In order to find the physical limits of the validity of the described method, multiple simulations are performed with the heat exchanger at $Y=50,25,10,8,7$ and $6 \mathrm{~mm}$, which correspond to $10 \mathrm{~L}$, $5 \mathrm{~L}, 2 \mathrm{~L}, 1.4 \mathrm{~L}$ and $1.2 \mathrm{~L}$. The network model approach is then taken and the time signals and Eq. (3) are used to obtain the TF and TM for the burner, heat exchanger and total system. In Fig. 12, some of the cases $(Y=10,8,7$ and $6 \mathrm{~mm})$ are shown as pairs of 2D plots of reaction heat (left side) and temperature (right side). Merging of the temperature fields of the flame and heat exchanger is visible for all except $Y=10 \mathrm{~mm}$, and flame quenching and impingement occur for $Y=7$ and $6 \mathrm{~mm}$. It is important to note that depending on the mixing and/or diffusivity of velocity, concentration and temperature, the heat exchanger may first be exposed to a non-flat profile associated to any of them. However, in lean premixed combustion, where thermoacoustic instabilities are important, the time scales of viscous dissipation is much larger than thermal and species diffusion and mixing. Therefore, the focus of the analysis is on the velocity profiles.

The gain and phase of the burner $T F$ for different distances between the burner and the heat exchanger are plotted in Fig. 13. As observed in Section 5.2, the burner $T F$ in the non-impinging cases does not significantly change as the distance decreases. The reduction in the overshoot of the gain is due to carbon monoxide formation around the heat exchanger and the fact that all reaction source terms are integrated in the whole domain. Therefore, the carbon monoxide to carbon dioxide conversion is also included and slightly alters the integrated reaction source term. These differences were completely removed for an adiabatic heat exchanger. For $Y=7 \mathrm{~mm}$, the flame stretches due to the flow distortion around the tube (see Fig. 12), causing a longer flame front and a larger phase. However, for $Y=6 \mathrm{~mm}$ the flame front moves toward the high velocity region between the adjacent tubes. The higher velocity decreases the convective traveling time of perturbations along the flame front and results in a slightly smaller phase compared to when $Y=7 \mathrm{~mm}$.

In general, changing the distance between the burner and heat exchanger marginally changes the flame TF. However, for the impinging cases, the heat exchanger is additionally exposed to unburnt mixture. This results in a different behavior, like a different phase shift of the heat exchanger TF. Therefore, it is crucial to investigate the response of the heat exchanger and total system as well.

Figure 14 shows the gain and phase of the heat exchanger TF when its excitation $\left(u_{\text {mid }}\right)$ is calculated using the burner TM in Eq. (3) and the inlet velocity $\left(u_{i n}\right)$. For all non-impinging cases
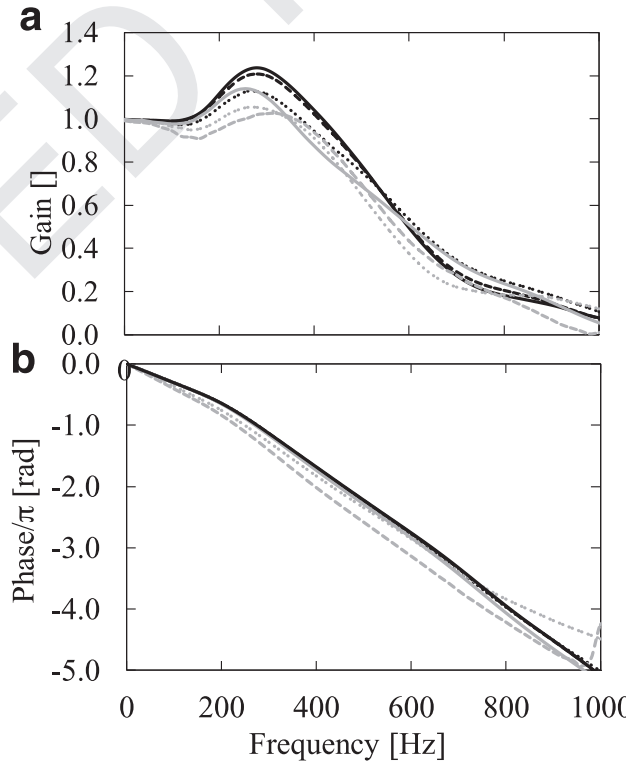

Fig. 13. The gain (a) and phase (b) of the burner $T F$ when the heat exchanger is at $Y=50$ (—), 25 (_-- ), 10 (.....), 8 (_), 7 (_--) and $6 \mathrm{~mm}(\ldots . .$.$) .$

$(Y=50,25,10$ and $8 \mathrm{~mm}$ ), the heat exchanger $T F$ is close to that 500 of the NoFlame case (see Fig. 11). For the impinging cases $(Y=7501$ and $6 \mathrm{~mm}$ ) however, a physical middle section between the burner 502 and heat exchanger does not exist and $u_{\text {mid }}$ in these cases is as- 503 sociated to a 'virtual' point. The extremely large gains for the im- 504 pinging cases are therefore an artifact of calculating using this vir- 505 tual point. In these cases, the heat exchanger is exposed to two 506 streams of hot (burnt) and cold (unburnt) flow (see the white and 507 black colors of the temperature contours in Fig. 12). As the veloc- 508 ity increases, the tube is further exposed to the cold stream and its 509 total heat flux decreases. This reflects in a phase shift of the order 510 of $\pi$ in the phase of the heat exchanger TF (see Fig. 14b).

For all the investigated cases, the mean heat flux at every point on the circumference of the heat exchanger tube is negative, i.e. in the absence of fluctuations, the heat exchanger is constantly absorbing heat from the system. However, for the impinging cases this sink is acting with a phase shift of $\pi$ (see Fig. 14b) and may have adverse effects. These effects should reflect in the dilatation rate and therefore the $T M$ of the total system. The gain and phase of the total TM are plotted in Fig. 15. The baseline is considered for the case without a heat exchanger (NoHex) and is marked by symbols. As intuitively expected, adding a heat exchanger far 


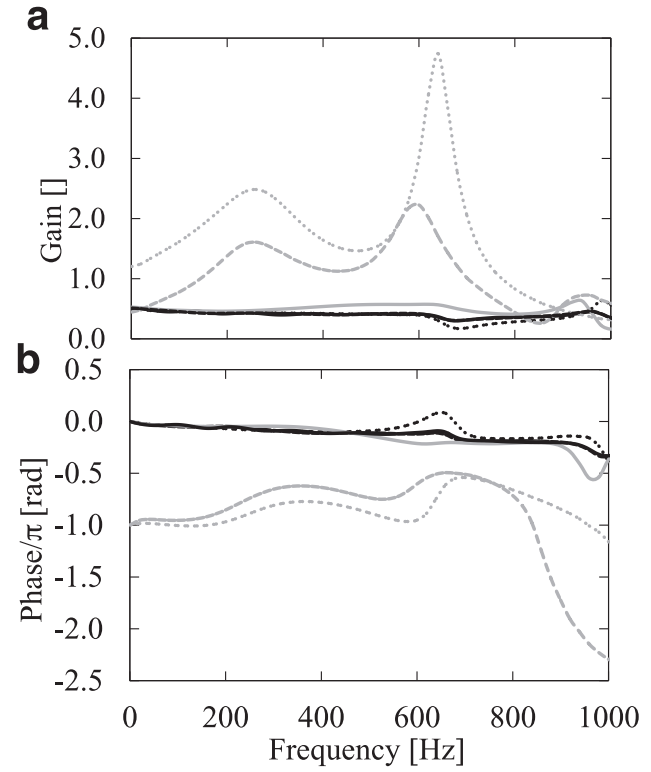

Fig. 14. The gain (a) and phase (b) of the heat exchanger $T F$ when it is placed at $Y=50\left(\_\right), 25(---), 10(\ldots \ldots), 8\left(\_\right), 7(\ldots--)$ and $6 \mathrm{~mm}(\ldots . .$.$) , and its ex-$ citation $\left(u_{\text {mid }}\right)$ is calculated using the burner TM in Eq. (3) and the inlet velocity $\left(u_{i n}\right)$.

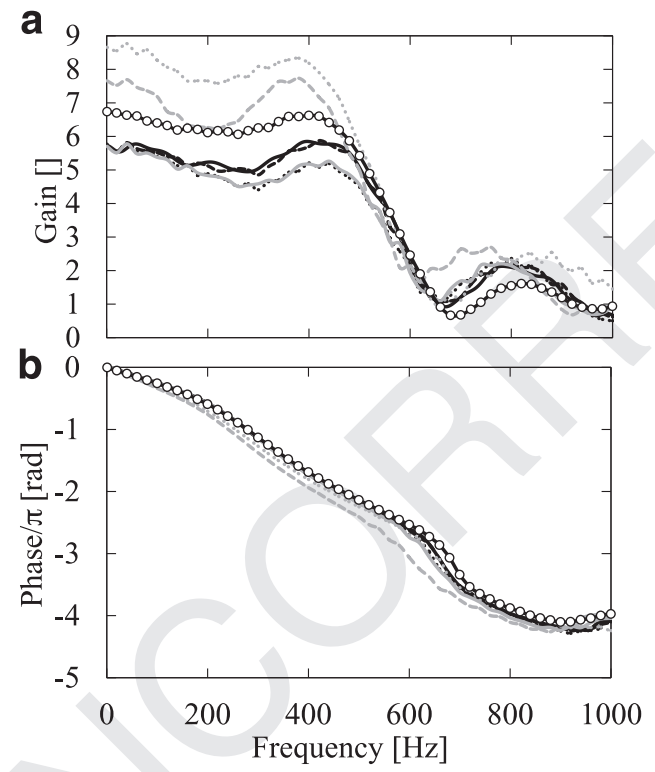

Fig. 15. The gain (a) and phase (b) of the total $T M$ in the NoHex case (-o-) and when the heat exchanger is placed at $Y=50$ ( $\left.\_\right), 25(-\ldots), 10(\ldots . .),. 8(-), 7$ $(-=-)$ and $6 \mathrm{~mm}(\ldots . .$.$) .$

downstream introduces an opposite temperature jump and decreases the gain of the total TM compared to the NoHex case. For the impinging cases on the other hand, the gain of the $T M$ is larger than that of the NoHex case. This is a result of the counter-phase behavior of the heat exchanger, i.e. although the total temperature ratio in Eq. (3) is smaller compared to the NoHex case, the gain of the total system TF is larger (see Fig. 7a), resulting in a larger TM and therefore larger dilatation rate.

Here a heat exchanger with only one tube row is considered and the results indicate that the phase of the heat exchanger has a negligible value compared to the burner. However, in other configurations such as multiple rows of heat exchanger tubes and the presence of von Kármán vortices, the heat exchanger may acquire larger phase values and adversely affect the instability of the sys- tem, even when placed far away from the burner [13]. In addition, 536 the methods and processes discussed in this work are applicable to 537 any other configuration of burners and heat exchangers. The gen- 538 eral conclusions to be used for this purpose are formulated in the 539 following section.

\section{Conclusions}

A method is introduced for predicting the thermoacoustic re- 542 sponse of a simplified heating appliance using the behavior of 543 its major constituting elements, i.e. the burner and the heat ex- 544 changer. To fulfill this purpose, the network model approach is 545 used to decouple the effects of the elements, considering details of 546 velocity profiles. The results of the conducted research allow for- 547 mulating the following conclusions:

- For a wide range of conditions, the TF/TM of the combination of the burner and heat exchanger can be represented as a superposition of their independently measured or modeled TF/TM's. A mathematical approach that allows the proper superposition of the individual TF/TM's into a combined TF/TM is proposed and validated.

- In the case of large distance between the burner and heat exchanger, the interactions are purely acoustic by nature and can be described within, e.g. the acoustic network model approach. The corresponding composition method provides good predictions for all distances between the burner and heat exchanger that are larger than the flame height. It shows that a flat velocity profile, that promotes planar acoustic waves, is not a necessity for decoupling the system using this method.

- For distances comparable to flame length, the method requires registering average velocities on a physical plane between the flame tip and the heat exchanger surface. This is possible for all distances longer than the flame length if there are negligible intrusions in temperature and/or species concentration profiles. If not, the interaction intensifies and causes the phenomenon of flame impingement.

- In general, in all cases without flame impingement, one can treat the burner and the heat exchanger as two acoustic elements acting opposite each other, i.e. the oscillations of dilatation rate introduced by the burner are reduced due to the presence of the heat exchanger.

- When the distance between the burner and heat exchanger is so small that flame impingement occurs, modifications of both the flame shape, temperature and the profile of flow approaching the heat exchanger takes place. In this case, the combination of the burner plus heat exchanger has to be considered as one coupled thermoacoustic element. Furthermore, the response of the coupled system in these cases changes drastically due to the heat exchanger acting as a counter-phase sink and increasing the cumulative oscillations of dilatation rate.

This investigation also implies that a correct modeling of the thermoacoustic response of a heating appliance requires the inclusion of the effects of both the burner and the heat exchanger as active acoustic elements, especially when flame impingement occurs. A heat exchanger placed relatively far downstream the burner results in a decrease in the gain of the combined $T M$, that may help abate possible instabilities. On the contrary, if flame impingement occurs the effects of the heat exchanger may enhance these instabilities.

\section{Acknowledgments}

The presented work is part of the Marie Curie Initial Train- 594 ing Network Thermo-acoustic and aero-acoustic nonlinearities in 595 green combustors with orifice structures (TANGO). We gratefully ,

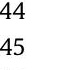

\section{7}


N. Hosseini et al./Combustion and Flame $x x x$ (2018) $x x x-x x x$

acknowledge the financial support from the European Commission under call FP7-PEOPLE-ITN-2012.

\section{References}

[1] L. Rayleigh, The explanation of certain acoustical phenomena, Nature 18 (1878) 319-321.

[2] B.D. Mugridge, Combustion driven oscillations, J. Sound Vib. 70 (1980) $437-$ 452.

[3] T. Poinsot, Prediction and control of combustion instabilities in real engines, Proc. Combust. Inst. 36 (2016) 1-28.

[4] S. Jaensch, M. Merk, E.A. Gopalakrishnan, S. Bomberg, T. Emmert, R.I. Sujith, W. Polifke, Hybrid CFD/low-order modeling of nonlinear thermoacoustic oscillations, Proc. Combust. Inst. 36 (2016) 3827-3834.

[5] W. Polifke, A. Poncet, C.O. Paschereit, K. Döbbeling, Reconstruction of acoustic transfer matrices by instationary computational fluid dynamics, J. Sound Vib. 245 (2001) 483-510.

[6] S. Jaensch, T. Emmert, C.F. Silva, W. Polifke, A grey-box identification approach for thermoacoustic network models, ASME Turbo Expo (2014) paper V04BT04A051.

[7] R. Rook, Acoustics in burner-stabilised flames, PhD thesis, Eindhoven University of Technology, 2001.

[8] E. Freitag, On the measurement and modelling of flame transfer functions at elevated pressure, PhD thesis, Technical University of Munich, 2009.

[9] S. Bomberg, T. Emmert, W. Polifke, Thermal versus acoustic response of velocity sensitive premixed flames, Proc. Combust. Inst. 35 (2015) 3185-3192.

[10] V. Kornilov, L.P.H. de Goey, Combustion thermo-acoustics in context of activitystability criteria for linear two-ports, 8th European Combustion Meeting (2017) paper ECM2017.0306.

[11] M.J. Lighthill, The response of laminar skin friction and heat transfer to fluctuations in the stream velocity, Proc. R. Soc. A Math. Phys. Eng. Sci. 224 (1954) $1-23$.

[12] A. Witte, W. Polifke, Modeling heat transfer and skin friction frequency response of a cylinder in pulsating cross-flow - a unifying perspective, 12th International Conference on Heat Transfer, Fluid Mechanics and Thermodynamics (2016) paper 1022.

[13] N. Hosseini, O.J. Teerling V. Kornilov, I. Lopez Arteaga, L.P.H. de Goey, Thermoacoustic instabilities in a Rijke tube with heatingcooling elements, 8th European Combustion Meeting (2017) paper ECM2017.0300.

[14] D. Durox, T. Schuller, S. Candel, Self-induced instability of a premixed jet flame impinging on a plate, Proc. Combust. Inst. 29 (2002) 69-75.

[15] T. Schuller, D. Durox, S. Candel, Dynamics of and noise radiated by a perturbed impinging premixed jet flame, Combust. Flame 128 (2002) 88-110.

[16] E.C. Fernandes, R.E. Leandro, Modeling and experimental validation of unsteady impinging flames, Combust. Flame 146 (2006) 674-686.

[17] K.S. Kedia, A.F. Ghoniem, An analytical model for the prediction of the dynamic response of premixed flames stabilized on a heat-conducting perforated plate, Proc. Combust. Inst. 34 (2013) 921-928.
[18] S. Schlimpert, M. Meinke, W. Schröder, Nonlinear analysis of an acoustically excited laminar premixed flame, Combust. Flame 163 (2016) 337-357.

[19] A. Albayrak, R.S. Blumenthal, A. Ulhaq, W. Polifke, An analytical model for the impulse response of laminar premixed flames to equivalence ratio perturbations, Proc. Combust. Inst. 36 (2016) 3725-3732.

[20] L. Strobio Chen, N. Hosseini, W. Polifke, O.J. Teerling, I. Lopez Arteaga, L.P.H. de Goey, Acoustic scattering behavior of a 2D flame with heat exchanger in crossflow, 23rd International Congress on Sound and Vibration (2016) paper 489.

[21] N. Hosseini, V. Kornilov, O.J. Teerling, I. Lopez Arteaga, L.P.H. de Goey, Development of a numerical model for obtaining flame transfer function in a simplified slit burner with heat exchanger, 21st International Congress on Sound and Vibration (2014) paper 279.

[22] F. Duchaine, F. Boudy, D. Durox, T. Poinsot, Sensitivity analysis of transfer functions of laminar flames, Combust. Flame 158 (2011) 2384-2394.

[23] ANSYS Fluent, Release 17.0, Help System, Species Transport and Finite-Rate Chemistry, ANSYS Inc., 2017.

[24] M. Manohar, Thermo-acoustics of Bunsen type premixed flames, PhD thesis, Eindhoven University of Technology, 2011.

[25] V. Kornilov, Experimental research of acoustically perturbed Bunsen flames, $\mathrm{PhD}$ thesis, Eindhoven University of Technology, 2006.

[26] V. Kornilov, K.R.A.M. Schreel, L.P.H. de Goey, Experimental transfer function of acoustically perturbed Bunsen-type flame, 2nd European Combustion Meeting (2005) paper 188.

[27] W. Polifke, C.O. Paschereit, D. Klaus, Constructive and destructive interference of acoustic and entropy waves in a premixed combustor with a choked exit Int. J. Acoust. Vib. 6 (2001) 135-146.

28] A. Huber, W. Polifke, Dynamics of practical premixed flames - part II: identification and interpretation of CFD data, Int. J. Spray Combust. Dyn. 1 (2009) 229-249.

[29] H.M. Heravi, A. Azarinfar, S.I. Kwon, P.J. Bowen, N. Syred, Determination of laminar flame thickness and burning velocity of methane-air mixtures, 3rd European Combustion Meeting (2007).

[30] V. Ratna Kishore, N. Duhan, M.R. Ravi, A. Ray, Measurement of adiabatic burning velocity in natural gas-like mixtures, Exp. Therm. Fluid Sci. 33 (2008) 10 16.

[31] M.J. Brown, C.J. Lawn, Dependence of the laminar burning velocity of methane propane and ethylene on initial temperature and inert diluent concentration, 1st European Combustion Meeting (2003).

[32] V. Kornilov, R. Rook, J. ten Thije Boonkkamp, L.P.H. de Goey, Experimental and numerical investigation of the acoustic response of multi-slit Bunsen burners Combust. Flame 156 (2009) 1957-1970.

[33] L.P.H. de Goey, L.M.T. Somers, W.M.M.L. Bosch, R.M.M. Mallens, Modeling of the small scale structure of flat burner-stabilized flames, Combust. Sci. Technol. 104 (1995) 387-400.

[34] S.W. Rienstra, A. Hirschberg, An Introduction to Acoustics, Lecture Notes, Eindhoven University of Technology, 2015.

[35] W. Polifke, A. Gentemann, Order and realisability of impulse response filters for accurate identification of acoustical multi-ports from transient CFD, Int. J. Acoust. Vib. 9 (2004) 139-148. 